\title{
Introducción a la traducción de textos ECOFIN de la UE: marcos teóricos, agentes y casos prácticos
}

\author{
(Introduction to the EU-ECOFIN texts translation: \\ theoretical frameworks, agents and case studies)
}

\author{
José María CAStelLano Martínez \\ jmcastellano@uloyola.es \\ Universidad Loyola Andalucía
}

Fecha de recepción: 5 de junio de 2015

Fecha de aceptación: 1 de julio de 2015

Resumen: El presente artículo presenta una propuesta de docencia en el ámbito de la traducción, interpretación y terminología de textos económicos y financieros de la Unión Europea, con especial atención al hecho multilingüe. En un principio se estudia la evolución histórica de la política multilingüe europea para a continuación ofrecer una propuesta triple a modo de base traductológica referencial constituida por un marco teórico, una relación de agentes participantes en esta terminología y, por último, determinados casos prácticos que arrojen luz a la labor del alumnado.

Palabras clave: Traducción institucional, Unión Europea, ECOFIN, Multilingüismo, Docencia, Terminología.

Abstract: This work presents a proposal of teaching in the field of translation, interpretation and terminology of economic and financial texts of the European Union paying special attention to the Multilingualism. Firstly, I study the historical development of the European multilingual policy. And after that, I offer a proposal based on a triple perspective which consists of a theoretical framework, a list of terminology-participant agents and, finally, some case studies that shed light on the work for the students.

Keywords: Institutional Translation, European Union, ECOFIN, Multilingualism, Teaching, Terminology.

INTRODUCCIÓN

El nacimiento de la UE es fruto de los distintos acontecimientos históricos que tienen lugar en Europa a lo largo de los siglos XX y XXI. Los conflictos bélicos que tienen lugar en los últimos ciento cincuenta años 
determinarán, de forma definitiva, un cambio de actitud de los Estados europeos, que desean la paz y la seguridad del viejo continente.

Así, en la segunda mitad del siglo XX Europa se encuentra asolada por las consecuencias de la II Guerra Mundial, el mayor conflicto bélico de la historia. La maltrecha situación económica y social de los países europeos impulsa el desarrollo de una tímida cooperación entre los Estados. El 9 de mayo de 1950, Robert Schumann, ministro francés de Asuntos Exteriores, propone llevar a cabo una política de cooperación común del carbón y el acero entre Francia y la República Federal Alemana, enemigos declarados en la mayoría de las guerras que han asolado Europa, incluidas las mundiales. A esta iniciativa se sumarían los países del Benelux y, en el último momento, Italia.

A partir de este momento se llevarán a cabo una serie de acciones y la firma de diversos tratados, que culminarán con la creación de la Unión Europea. Se puede decir, por lo tanto, que el proyecto de construcción europea viene determinado por la necesidad de recuperación económica que azotaba al Viejo Continente de postguerra. Otrora, fueron las dinastías, las religiones o las artes las que potenciaron una unión del continente, sin embargo fue la Economía la que materializó como ningún otro medio un marco jurídico e institucional común para 28 Estados europeos.

Con el impulso de unión económica y comercial así como con la incorporación del Reglamento 1/1958, se inicia un proceso de unificación normativa y terminológica que quedará reflejado a lo largo de los años en la producción documental de las instituciones europeas. Ahora bien, la cuestión que aquí nos interesa se centra en la terminología económica y financiera (ECOFIN) surgida y compartida por parte de las instituciones europeas y extrapoladas a los Estados miembros en el ámbito legislativo, institucional y también social y comunicativo.

Existen ya ciertos trabajos con respecto a la traductología y terminología en el seno de las instituciones europeas, no obstante la ausencia de marco teórico propio para este tipo de traducción plantea un vasto ámbito de investigación en donde confluyen factores muy diversos que deberán tenerse en cuenta más allá de lo lingüístico, como también lo político, lo jurídico, lo cultural o lo comunicativo.

Resulta pertinente también que tanto el público receptor diferencie claramente las instituciones y organismos que participan de esta terminología ECOFIN europea, la cual se ve en ocasiones afectada por el tratamiento informativo de los medios. Este hecho provoca confusión y ambigüedad que deberá combatirse para asegurar el buen conocimiento de los términos, el origen y motivo de los mismos así como sus equivalencias en las lenguas oficiales. 
Por ello, se ofrece a continuación una propuesta docente en materia de traducción (y por afinidad también para la traducción y terminología) del ámbito ECOFIN europeo en el que se diferencian tres estadios que pretenden conformar una base traductológica que sirva de marco referencial al alumnado. En primer lugar se aborda la cuestión del multilingüismo, sus orígenes y finalidad, para a continuación ofrecer un marco teórico con especial mención a la equivalencia y a la recepción del mensaje. En este apartado destacan las aportaciones de autores que centran su trabajo en la propia Unión Europea.

Una vez superado el "aparato teórico", se procede a establecer una relación de agentes que intervienen en la dinámica económica y financiera de la Unión Europea y por tanto en la generación de términos ECOFIN. EI alumnado debe manejar esta terminología así como saber diferenciar los conceptos de manera eficiente de tal modo que sepa identificar a las partes protagonistas de esta materia.

Por último se ofrece un bloque más práctico en torno a la cuestión de la armonización terminológica ECOFIN con especial énfasis en las siglas y la concreción conceptual que representan. Se ofrece, pues, una propuesta docente fundamentada en un marco teórico, unos agentes productores y participativos así como casos prácticos que pretenden constituir una base referencial para el alumnado de esta materia.

\section{EL MULTILINGÜISMO OFICIAL COMO HITO HISTÓRICO: EL REGLAMENTO 1/1958}

La diversidad de lenguas y culturas que integra la sociedad europea de finales del siglo XX y principios del XXI se refleja, en buena medida, en la política lingüística actual de la Unión Europea: el multilingüismo. Éste aboga por la oficialidad de 24 lenguas, estableciendo un contexto único por el cual las instituciones comunitarias precisan de la traducción y de la interpretación como labores indispensables que posibilitan la comunicación, por un lado, entre la Unión y la ciudadanía; por otro, la comunicación inter- e intrainstitucional. En este sentido, R. Goffin afirma:

\footnotetext{
Jamais on n'a autant confronté et contrasté, en situation concrète de communication, les ressources multiples de langues. Jamais on n'a autant légiféré, jamais on n'a eu l'occasion d'étudier au quotidien comment des institutions internationales à vocation juridique agissent sur le plan langagier pour décrire et nommer des notions nouvelles dans le choc de neuf langues naturelles, enracinées dans leurs traditions juridiques, économiques, culturelles, sociales et politiques (1994: 636).
}

La construcción de la Unión Europea ha creado y desarrollado un aparato institucional que repercute en las legislaciones y sistemas jurídicos 
de los Estados miembros. Este fenómeno únicamente ha sido posible por medio de la lengua y de la traducción; proceso en el que se ha generado terminología multilingüe en virtud de las materias sobre las que Estados miembros e instituciones europeas legislan. De ahí que el sector económico y financiero (ECOFIN), también monetario y comercial, haya cobrado tal relevancia en la Unión.

El marco jurídico en el que surge y se desarrolla la política multilingüe comunitaria encuentra su principio inmediato en el artículo 190 del Tratado constitutivo de la Comunidad Europea de la Energía Atómica (CEEA EURATOM): "El régimen lingüístico de las instituciones de la Comunidad será fijado por el Consejo, por unanimidad, sin prejuicio de las disposiciones previstas en el reglamento del Tribunal de Justicia". De este modo, se otorga al Consejo autoridad para establecer el régimen lingüístico comunitario.

El Reglamento 1/1958 de este órgano reconoce en el art. 1 que "las lenguas oficiales y las lenguas de trabajo de las instituciones de la Comunidad serán el alemán, el francés, el italiano y el neerlandés", aunque, hasta llegar a la actual cifra de 24 , este articulado se ha visto modificado conforme se han adherido nuevos Estados a las antiguas Comunidades Europeas y actual Unión.

El carácter oficial otorga a cada una de las lenguas la misma validez y autenticidad en cualquier texto, escrito $u$ oral, de las instituciones de la Unión. Se perfila, por tanto, un contexto multilingüe donde los servicios lingüísticos ostentarán una función protagonista: "Aborder el multilinguisme et le problème de l'équivalence des termes conduit à ressentir davantage le besoin d'une prise en compte du fait polysémique" (Janssen y Van Campenhoudt, 2005: 64).

La primera piedra del edificio del multilingüismo institucional proviene de la búsqueda de la "equivalencia" de la validez y autenticidad de los documentos que integran la jurisprudencia de la Comunidad Europea del Carbón y el Acero (CECA) en todas sus versiones existentes. Sobre esta cuestión, C. Heynold afirma lo siguiente:

La première organisation européenne, à savoir la Communauté européenne du charbon et de l'acier (CECA), créée en 1951, a privilégié le français (...). La mise en place de la Cour d'arbitrage prévue par le Traité de Paris a fait surgir la nécessité d'assurer une équivalence de la jurisprudence dans les quatre langues (le français, l'allemand, l'italien et le néerlandais) des six pays membres de la CECA. Ainsi, dès le démarrage de la première communauté, il est devenu évident qu'afin que nul n'ignore la loi, il n'est pas posible d'imposer celle-ci dans une langue étrangère. 
Les pères fondateurs de la deuxième organisation européenne, la Communauté économique européenne (CEE), en ont tiré l'enseignement qu'il valait mieux rédiger tout de suite le texte qui devait régir son fonctionnement, c'est-à-dire le Traité de Rome, dans les quatre langues officielles, chaque versión faisant également foi (article 247) (1999: 5).

El motivo por el que surge la política del multilingüismo en el seno de las primeras instituciones comunitarias se debe, por lo tanto, a la cuestión de la "equivalencia" necesaria para la incorporación de la normativa comunitaria en las legislaciones de los Estados miembros.

Es cierto que el francés siguió siendo durante los siguientes años la lingua franca de las Comunidades Europeas y que quizás sea temprano aún para hablar propiamente de multilingüismo, pues más bien se trataba de una situación de plurilingüismo, en la que la mayoría de los funcionarios conocían las cuatro lenguas oficiales y trabajaban en temáticas muy limitadas, siendo mucho más fácil especializarse en un ámbito concreto, dominar la terminología e incluso expresarse en varias de las lenguas oficiales (Brackeniers, 1995: 14).

\section{PRopuesta de MARCo teÓRICO APLICABLE}

La docencia en traducción, interpretación y terminología encuentra marco teórico inmediato en las teorías tradicionales sobre equivalencia que surgieron a mediados del pasado siglo. En especial, considero que las siguientes obras son esenciales en tanto que punto de partida en cualquier materia traductológica: por una parte, el estudio del concepto de equivalencia en traducción que se desprende del ensayo On linguistic Aspects of Translation de R. Jakobson (1959); por otra, la obra Toward a Science of Translating with Special Reference to Principles and Procedures involved in Bible Translation de E. Nida (1964).

La primera de ellas aborda la cuestión de la equivalencia en traducción desde una perspectiva lingüística, afirmando el autor que ésta no existe. En todo caso, prefiere hablar de "equivalencia parcial", ya que según el autor, no se traducen unidades lingüísticas sino ideas, conceptos o mensajes. En lo que respecta a la traducción de un texto, R. Jackobson considera que en sí se traduce el mensaje del texto a una nueva lengua, por lo que cualquier mensaje se podría traducir a cualquier idioma, en tanto que cada uno de ellos posea los mecanismos pertinentes para representarlo.

La segunda de ellas supone un antes y un después en la Traductología. Si Jakobson proponía un enfoque de carácter lingüístico para abordar esta cuestión, Nida propone un enfoque comunicativo mediante el cual definirá al proceso traductor como el acto de reproducir en el TM una equivalencia que sea lo más fiel posible al TO y que se caracteriza por 
respetar, en primer lugar, el contenido y, en segundo, el estilo. Es posible distinguir, por lo tanto, dos tipos de equivalencia: una equivalencia formal -aquella que es fruto de una traducción más literal en la que prima mantener con escrúpulo la forma del TO- y una equivalencia dinámica o funcional -aquella en la que es más importante transmitir la idea expresada en el TO de acuerdo con las particularidades de la lengua del TM.

En consonancia con las posturas anteriores, siempre en el marco del sentido de la equivalencia dinámica o funcional, P. Newmark (1988) recurrirá a la idea de "equivalencia cultural" como posible estrategia de traducción consistente en sustituir un elemento cultural del TO por otro que consiga un efecto equivalente en el TM, en armonía con la lengua y cultura de llegada.

Esta introducción teórica es recomendable y pertinente en tanto que el alumnado debe asumir que la traducción, la interpretación y la terminología, ya sea técnica 0 arte, se encuentran potencialmente vinculadas a lo lingüístico, a lo comunicativo y a lo cultural, en donde el concepto de equivalencia no deja de ser una quimera que siempre suscitará debate y dilemas dependiendo del texto y del contexto en definitiva.

No obstante, los marcos teóricos anteriores resultan sólo introductorios para esta materia aunque insuficientes. En el ámbito europeo coexisten otros factores condicionantes en el acto de traducción: el acervo comunitario, que vincula jurídicamente a las diferentes versiones lingüísticas de los textos oficiales, así como también los contextos políticos, sociales y comunicativos.

Los estudios realizados sobre el concepto de equivalencia y la traducción en el ámbito institucional de la Unión Europea no son muy frecuentes, aunque si bien interesantes. Por lo general, son trabajos relativamente recientes $\mathrm{y}$ de alto contenido pragmático, por ello que es conveniente incorporarlos en el aparato teórico de la docencia en traducción, interpretación y terminología.

Las particularidades del ámbito europeo quedan introducidas por $\mathrm{S}$. Šarcevic quien afirma que el principio de fidelidad del TO se devalúa progresivamente en favor del principio de fidelidad del texto, documento, objeto o instrumento en cuestión. Dicho de otro modo: el propio contexto institucional europeo "degrada" la presencia del TO a favor del instrumento jurídico o institucional que éste representa, pues, en tanto que todas las versiones lingüísticas son apriorísticamente equivalentes, la noción de TO desaparece en tanto que lo que importa es la existencia de ese mismo instrumento en cada una de las lenguas oficiales.

Existen otras valoraciones en el ámbito traductológico de la Unión Europea, por ejemplo, el concepto de "equivalencia existencial" que plantea K. Koskinen. Según la autora finlandesa, la traducción a nivel europeo no se 
contempla como un acto de comunicación, sino más bien como acto simbólico que refleja el principio del multilingüismo. Además, adjetiva a este tipo de comunicación como "acultural", ya que no se localiza a priori un TO, lo cual contraviene las especificidades culturales de cada lengua y sociedad en pro del Multilingüismo. Asimismo, en consonancia con K. Koskinen, T. J. M. Van Elsadmite que la simplificación del contenido y la sintaxis de los textos institucionales europeos implica una "desculturalización", que se aproxima, aunque con matices muchos más negativos, al carácter "acultural" anterior.

Llegados a este punto, tanto Koskinen como Šarcevic aseguran que la relación textual más importante en la traducción institucional de la Unión Europea no se da entre el TO y el TM, sino entre los textos individuales vistos desde el contexto institucional meros instrumentos.

Sin embargo, la postura más novedosa, así como reciente, es la de A. L. Kjær,quien afirma que en la Unión Europea no existe traducción como tal, sino más bien una reproducción textual interlingüística, ya que la finalidad principal del denominado acto traductor a nivel comunitario pretende, ante todo, recrear en todas las lenguas oficiales un texto simétrico que asegure la cohesión del mismo en todos los idiomas sin prestar atención a las particularidades y características de cada una de ellas. Se desvanece, pues, el propio concepto convencional de traducción.

El panorama aquí abordado, uno general y el otro más sectorial, constituye un marco teórico fundamental en la docencia de materias o formación relacionada con la traducción, la interpretación y la terminología de documentos de la Unión Europea. Una vez se ha aclarado el contexto de trabajo, resulta pertinente continuar con los agentes que intervienen en los textos ECOFIN europeos.

\section{AGENTES EUROPEOS ECONÓMICOS Y FINANCIEROS: LUCES Y SOMBRAS}

La docencia en traducción, interpretación y terminología implica mucho más que el mero conocimiento lingüístico, en especial en textos especializados en donde las interferencias de lengua, materias y marco son mucho más exigentes. El caso de los textos relacionados con el ámbito económico y financiero europeo reclama, además, especial atención con respecto a las fuentes y los términos del TO y TM en tanto que se corresponden a un marco jurídico e institucional armonizado, legislado y multilingüe.

Sin menoscabo de la existencia de términos económicos o financieros equivalentes en otras lenguas y ámbitos, la terminología ECOFIN europea presenta particularidades en tanto que al estar íntimamente relacionada con el poder político e institucional de los Estados miembros, aquélla sufre la exposición mediática. Ante tal caudal informativo, el alumnado suele incurrir 
en confusión con respecto a la terminología así como también a los organismos europeos que participan en la creación ECOFIN de la Unión.

A continuación se indican cuáles son los principales organismos o entes europeos, sus equivalencias y funciones, las cuales deberán explicarse y exponerse al alumnado con especial énfasis en la fase documental de la traducción de textos de este tipo, pues el proceso de documentación deberá contar fundamentalmente con la presencia del organismo fuente. Si el alumnado desconoce el funcionamiento y el significado de esta sopa de siglas y denominaciones, afrontar el texto en cuestión implicará una mayor complejidad conceptual.

[ES] Consejo de Asuntos Económicos y Financieros (Ecofín)

Definición, funciones: Constituido por los Ministros de Economía y Hacienda de todos los Estados miembros. Los Comisarios Europeos competentes también participan en las sesiones.

Por lo general las reuniones del Ecofín se celebran una vez al mes. Coordinar las posiciones de la UE en reuniones internacionales, como el G20, el Fondo Monetario Internacional y el Banco Mundial.

[EN] Economic and Financial Affairs Council configuration (Ecofin)

[FR] Conseil "Affaires économiques et financières" (ECOFIN)

[DE] Rat "Wirtschaft und Finanzen" (Rat ECOFIN)

[ES] Eurogrupo

Definición, funciones: Órgano informal que reúne a los ministros de la zona euro. Asegurar estrecha coordinación entre las políticas económicas de los Estados miembros de la zona del euro. Preparar la Cumbre del Euro y de los correspondientes trabajos consecutivos. Se reúne normalmente una vez al mes, la víspera de la sesión del Consejo de Asuntos Económicos y Financieros.

[EN]Eurogroup

[FR]Eurogrupe

[DE]Euro-Gruppe

[ES] zona euro (eurozona)

Definición, funciones: Conjunto de Estados miembros de la Unión Europea que han adquirido como divisa oficial el euro. Los Estados de la zona euro son: Alemania, Austria, Bélgica, Chipre, Eslovaquia, Eslovenia, España, Estonia, Finlandia, Francia, Grecia, Irlanda, Italia, Letonia, Lituania, Luxemburgo, Malta, Países Bajos y Portugal

[EN] eurozone (euroarea)

[FR] zone euro (eurozone) 
[DE] eurozone (Euro-Währungsgebiet, Euroraum, Euro-19)

Nota: Según la terminología oficial del Banco Central Europeo y del Libro de estilo interinstitucional de la Unión Europea, para referirse al conjunto de naciones que participan en la moneda única conviene emplear la expresión zona del euro. En los medios de comunicación es frecuente encontrar también las formas zona euro y eurozona, de amplio uso y que, pese a no ser las denominaciones recomendadas por la UE para sus documentos, pueden considerarse asimismo adecuadas en el ámbito periodístico. Se recomienda escribir todas ellas con minúscula ya que no se trata de topónimos ni de nombres propios de una entidad política o institución. (Fundéu, 2009).

[ES] Eurosistema

Definición, funciones: Autoridad monetaria de la zona del euro constituida por el BCE y los bancos centrales nacionales de los Estados miembros cuya moneda es el euro. Su objetivo primordial es mantener la estabilidad de precios.

[EN] Eurosystem

[FR] Eurosystème

[DE] Eurosystems

Nota: El Sistema Europeo de Bancos Centrales (SEBC) está formado por el BCE y los bancos centrales nacionales de todos los Estados miembros de la Unión Europea (UE), hayan adoptado el euro o no. Por ello, el Eurosistema y el SEBC seguirán coexistiendo mientras haya Estados miembros de la UE que no pertenezcan a la zona del euro.

[ES] Banco Central Europeo (BCE)

Definición, funciones: $\mathrm{El} B C E$ es el banco central de la moneda única europea, el euro. Su función principal consiste en mantener el poder adquisitivo del euro y, con ello, la estabilidad de precios en la zona del euro.

[EN] European Central Bank (ECB)

[FR] Banque centrale européenne (BCE)

[DE] Europäischen Zentralbank (EZB)

[ES] Fondo Europeo de Estabilidad Financiera (FEEF)

Definición, funciones: entidad jurídica creada con carácter temporal por los Estados miembros de la eurozona en 2010 como mecanismo de resolución de crisis que asistiera a Irlanda, Portugal y Grecia en lo financiero.

[EN] European Financial Stability Facility (EFSF)

[FR] Fondseuropéen de stabilité financière (FESF) 
[DE] Europäische Finanzstabilisierungsfazilität (EFSF)

Nota: Existe otra variante del FEEF que responde a "Facilidad Europea de Estabilidad Financiera".

[ES] Mecanismo Europeo de Estabilización Financiera (MEEF)

Definición, funciones:mecanismo de rescate permanente que inicia sus operaciones en 2012 para responder a nuevas solicitudes de asistencia financiera a los Estados miembros de la zona euro. Ha sido el mecanismo que ha realizado préstamos financieros a España o Chipre.

[EN] European Financial Stabilisation Mechanism (EFSM)

[FR] Mécanisme européen de stabilisation financière (MESF)

[DE] Europäischer Finanzstabilisierungsmechanismus

[ES] Mecanismo Europeo de Estabilidad (MEDE)

Definición, funciones: Mecanismo permanente de resolución de crisis económicas para países de la zona euro. Su finalidad es movilizar fondos y proporcionar apoyo a la estabilidad financiera, bajo una estricta condicionalidad, a los miembros del MEDE que experimenten o corran el riesgo de experimentar graves problemas de financiación, cuando ello sea indispensable para salvaguardar la estabilidad financiera de la zona euro en su conjunto o de sus Estados Miembros. Con este fin, el MEDE podrá obtener fondos a través de la emisión de instrumentos financieros o mediante la celebración de acuerdos con sus propios miembros, entidades financiera y terceros.

[EN] European Stability Mechanism (ESM)

[FR] Mécanisme européen de stabilité (MES)

[DE] Europäische Stabilitätsmechanismus (ESM)

El conocimiento y manejo de los términos anteriores son fundamentales en la coyuntura actual en tanto que estos agentes económicos y financieros participan activamente en la producción de información mediática debido a su capacidad jurídica e institucional, la cual vincula a las legislaciones nacionales y por tanto a sus sociedades.

Por lo tanto y llegados a este punto, se han de tener en consideración: por una parte, el marco teórico anterior en el que se postulan modelos generales y específicos para este tipo de textos; por otra, la correcta diferenciación de los principales agentes que participan en la elaboración terminológica y textual, conociendo sus equivalencias lingüísticas en las lenguas de trabajo habituales. El siguiente estadio de esta propuesta se centra en conocer cuestiones prácticas de las que aprender, que permitan al alumnado adquirir conocimientos adicionales a la 
mera teoría. Con ello, se pretende ofrecer casos reales que, a modo de anécdotas o lecturas prácticas, refuerce la adquisición de sus competencias y destrezas, dote a su trabajo de referentes y enriquezca igualmente el aprendizaje.

\section{ALGUNOS CASOS PRÁCTICOS: ROCES Y FRICCIONES EN EL ÁMBITO ECOFIN}

Una vez se establece un marco teórico así como se identifican a los principales agentes que intervienen en el ámbito ECOFIN considero pertinente ofrecer al alumnado una batería de casos prácticos o ejemplos relevantes que contribuyan a consolidar la base traductológica con la que hacer frente a esta especialización.

4.1. El caso de la traducción del acervo comunitario al finés: la lengua inglesa propone pero el traductor dispone

En 1995, Finlandia pasó a ser Estado miembro junto con Suecia y Austria, abandonando automáticamente la Asociación Europea de Libre Comercio (AELC, en inglés EFTA), de la que era miembro desde 1985. No obstante, ya desde 1990 el Ministerio de Justicia finlandés comenzó a traducir al finés toda la documentación relativa al acervo comunitario tomando como referencia las versiones en lengua inglesa.

Este hecho supuso que entre 1992 y 1993, el Ministerio cambiara de criterio y comenzara de nuevo la traducción del acervo comunitario al finés aunque en esta ocasión tomando como TO las versiones en lengua francesa. El motivo fue, según Y. Gambier (1998: 296) que en tanto que la mayoría de estos textos en versión inglesa (tratados, reglamentos fundamentales, etc.) eran a su vez traducciones de las versiones francesas, resultaría más eficiente y efectivo tomar como TO las versiones lingüísticas en francés para evitar posibles ilegibilidades o malas interpretaciones.

En este sentido, S. Šarcevic destaca la preponderancia de la lengua inglesa en los países de la Europa oriental y los escandinavos:

Like Croatia, it appears that most of the applicant states from Central Eastern Europe (CEE) have selected English as the primary source text for translations of the acquis. As in the Scandinavian countries, English has become by far the dominant foreign language in these countries. In the interest of guaranteeing reliability, certain factors should be taken into account when determining how many and which texts should be consulted in addition to the English authentic texts. Above all, it is necessary to keep in mind that the English authentic texts are translations in the majority of cases (...) (2001: 41s.).

Por tanto, la conclusión principal que debemos extraer del caso finlandés será la importancia que tienen las fuentes documentales primarias 
frente a lo cuantitativo. Cierto es que la lengua inglesa ha devenido lingua franca internacional, incluso dentro la multilingüe Unión Europea, sin embargo, el ministerio finlandés fue consciente de las controversias que supondrían para futuras cuestiones interpretativas, legislativas, jurídicas o judiciales traducir desde la versión inglesa, que era a su vez una traducción de las versiones originales y en definitiva de la versión francesa.

\subsection{La moda de la "siglomanía": la anécdota del "ecu"}

Las organizaciones internacionales han contribuido a la proliferación de siglas y acrónimos para representar por medio de una grafía breve un contenido especializado. La Unión Europea no es una organización internacional al uso, más bien es una comunidad jurídica, política e institucional, aunque no por ello deja de recurrir al uso de siglas y acrónimos en sus textos. El ámbito ECOFIN no queda al margen, de ahí que R. Goffin afirme que las siglas proliferan para nombrar programas, acciones y sistemas:

Véritable culte, la siglomanie règne souveraine. Acronymes et autres sigles prolifèrent pour désigner programmes, actions et systèmes. Souvent évocateurs et mnémo-techniques, les abrègements contribuent largement à donner aux textes leur caractère cryptique (...) ou les acronymes leur caractères syllabique (...). Les services administratifs font preuve ici d'une belle inventivité nourriée de réminiscences mythologiques (1994: 60).

Como bien indicar el autor, la presencia excesiva de siglas o acrónimos en textos conlleva un mensaje encriptado, que escapa a la comprensión del público general y fomenta una "jerga" exclusiva para especialistas o profesionales de la materia en cuestión. Por ello que el abuso de siglas o acrónimos puede suponer un verdadero problema traductológico en caso de no existir un previo trabajo de armonización o equivalencias en las diferentes lenguas oficiales.

Cierto es que las sinergias entre los servicios lingüísticos de los organismos de la Unión Europea han ido en aumento. Prueba de ello es la convergencia de las diferentes bases de datos terminológicas que existían en cada institución europea hacia la actual IATE (Inter-Active Terminology for Europe). De ahí que EUTERPE (Parlamento Europeo) o TIS (Consejo de la UE) se integraran, entre otras, en EURODICAUTOM y éste a su vez se mejorase en la actual IATE (Castellano Martínez, 2014: 39-73).

Ejemplos como los siguientes evidencian la criptografía que en ocasiones puede suponer enfrentar a la traducción de textos o documentos 
europeos del ámbito ECOFIN: FEEF, MEEF, MEDE, SEBC, BCE, BEI, BUDG (Comisión presupuestaria del PE), CEE, CECA, CdE (Comité de las Regiones), CE, PE, CESE (Consejo Económico y Social Europeo), Coreper (Comisión de representantes permanentes), EcoFin, EEE (Espacio Económico Europeo), NACE (Nomenclature statistique des activités économiques dans la Communauté européenne), ZEE (zona económica exclusiva), CAFA (Comisión de Asuntos Financieros y Administrativos del Comité de las Regiones de la UE), etc.

En este sentido, J.P. Mingansson (1989: 65-66) presta especial mención a la forma escrita del acrónimo "Ecu" y critica la falta de armonización que existió a lo largo de su vigencia económica (1979-1998). El autor afirma que pese a la madurez financiera, que en su momento, tuvo la divisa de las Comunidades Europeas, la Comisión mantuvo la forma ECU en inglés, mientras que para el francés Ecu (sic). Estas formas supusieron el punto de partida para el resto de lengua oficiales del momento aunque sin que ninguna de ellas se usaran de forma homogénea ya que a nivel institucional, e incluso periodístico, cada lengua mantenía una u otra forma. En español, por ejemplo: ECU, Ecu o ecu.

Asimismo, el autor añade que el "Ecu InterserviceGroup" estimaba oportuno mantener en inglés la forma ecu y que la forma natural en francés debería ser écu, para corresponderse así con el resto de formas escritas de las principales divisas del momento (dólar/dollar/Dollar, libra esterlina/pound sterling/libre sterling/Pfund sterling o yen/Yen).

El autor critica la falta de voluntad o previsión de la Comisión al no proporcionar una forma fija en las lenguas oficiales del momento, ya que a finales de la década de los ochenta del pasado siglo eran varias las formas existentes en cada idioma: the Ecu (Financial Times) o l'ECU (Le Monde). Así, finaliza esta crítica con la propuesta (a nivel gráfico y fonético) de formas "armonizadas" para cada una de las lenguas oficiales de aquel entonces que el anterior grupo recomienda tal y como se indica en la siguiente tabla:

\begin{tabular}{|c|c|c|}
\hline Lengua & Singular & Plural \\
\hline DA (danés) & Ecuen & ecuerne \\
\hline DE (alemán) & der Ecu & die Ecu \\
\hline EL (griego) & to Ecu & ta Ecu \\
\hline EN (inglés) & the ecu & the ecus \\
\hline ES (español) & el ecu & los ecus \\
\hline FR (francés) & l'écu & les écus \\
\hline IT (italiano) & l'ecu & gli ecu \\
\hline NL (neerlandés) & de ecu & de ecu's \\
\hline
\end{tabular}




\begin{tabular}{|l|l|l|}
\hline PT (portugués) & o ecu & os ecus \\
\hline
\end{tabular}

Por último, recomienda también utilizar la forma "ECU" como sigla en consonancia con el resto de divisas de aquel entonces: USD (dólar), FF (franco francés), HFL (florín holandés), etc. En cuanto al signo gráfico o extralingüístico se prefirió la forma $\mathrm{E}$. No hace falta advertir que con la llegada del Euro $(€)$, divisa actual que mantiene forma normalizada en cada lengua oficial así como un signo (€) y una sigla (EUR), el ecu dejó de existir.

Este caso evidencia la importancia que tiene la armonización de términos, también de sus siglas y acrónimos, en contextos fuertemente institucionalizados y multilingües cuyos contenidos vinculan jurídica y económicamente a personas físicas y jurídicas. Por ello, la labor traductora de documentos europeos ECOFIN debe acudir a fuentes contrastadas y oficiales, así como también rehuir de traducir motu proprio cualquier tipo de término, sigla o acrónimo ya que se corre peligro de desvirtuar el TO o remitir a conceptos o entidades distintas o inexistentes.

\subsection{Armonizar para traducir: los términos ECOFIN en directo}

A tenor del apartado anterior, retomando la cuestión de la armonización terminológica en la Unión Europea, hay que destacar que el contexto de crisis económica y financiera ha impulsado el surgimiento de nuevos conceptos ECOFIN que se caracterizan por ser variables, cambiantes en poco tiempo y sin referentes naturales en las LM en cuestión.

El advenimiento de instrumentos, operaciones y mecanismos financieros que hicieran frente a la hecatombe económica en la que se sumían nuestras economías europeas (y se sumen, aunque con menos alarmismo mediático) ha evidenciado la ausencia de procedimientos de armonización regulados o estables que proporcionaran una misma terminología común y equivalente entre todas las lenguas oficiales. Así lo evidencia L. González que analiza determinados instrumentos relacionados con la denominada "deuda estructurada" contemplados en el Reglamento Delegado (UE) n.. 446/2012 destacando de ellos la preponderancia de las siglas inglesas en otras lenguas como la española, la preferencia con una matriz común para términos aproximados o nuevos y la biunivocidad de la traducción de determinados términos de la lengua inglesa en la mayoría del resto de lenguas oficiales.

El principal interés que L. González (2012: 2) muestra en su trabajo, y he aquí la relevancia de este apartado, es la armonización de una terminología transparente y estable. Frente a la ausencia de "soluciones más drásticas", como indica el autor, los procedimientos y las operaciones financieras surgidas de la burbuja especulativa resultan opacas y difusas: 
La terminología que utilizamos para referirnos a estos instrumentos es también, en consecuencia, compleja, modular y, a menudo, opaca porque los términos se acuñan en inglés y, en el mejor de los casos, se traducen a las demás lenguas.

Asimismo, el autor indica que en lenguas como el italiano o el neerlandés estos términos no se traducen mientras que en español sí, pero manteniéndose las siglas en inglés. En este sentido, el L. González ofrece un análisis detallado de términos ECOFIN del que podemos destacar en especial collateralized debt obligation (CDO), que en español es "obligación garantizada por deuda". Este término es el punto de partida para otros términos ECOFIN recogidos en el citado reglamento como son:

- collateralized bond obligation (CBO): obligación garantizada por bonos (CBO),

- collateralized fund obligation (CFO): obligación garantizada por fondos (CFO),

- collateralized mortgage obligation (CMO): obligación garantizada por hipotecas (CMO),

- collateralized loan obligation (CLO): obligación garantizada por préstamos (CLO).

Lo anterior evidencia que la necesidad multilingüe, que se materializa a través de la traducción, adolece de fórmulas que armonicen la realidad conceptual ECOFIN sobrevenida en los últimos años a todas las lenguas oficiales, bien por la ausencia de decisiones drásticas que anunciaba el autor, bien por la opacidad y falta de limitación conceptual que muchos de estos instrumentos o procedimientos tienen.

La labor de L. González, incipiente en la materia, se complementa con el glosario inglés-español de términos y expresiones sobre la crisis económica que el Grupo de coordinación, calidad y terminología del departamento de lengua española en la Dirección General de Traducción de la Comisión Europea propone en el mismo volumen (2012: 9-14) elabora indicando además el código IATE de la unidad terminológica en cuestión.

En resumen, este caso debe aportar al alumnado la importancia que tiene el uso de una terminología armonizada en un sector como es el ECOFIN. Continuamos, pues, las cuestiones ya planteadas en el apartado anterior aunque en esta ocasión con la ilustración de términos recientes surgidos de la especulación económica y su repercusión a nivel europeo. 


\section{CONCLUSIONES}

La propuesta docente aquí presentada responde a la necesidad de ofrecer al alumnado de traducción, interpretación y terminología una base traductológica referencial en la materia ECOFIN europea. Por ello que este trabajo ha abordado tres estadios bien diferenciados: el teórico, el agente y el práctico. Tras los apartados anteriores, se puede concluir el siguiente esquema conceptual con el que representar el contenido de este artículo:

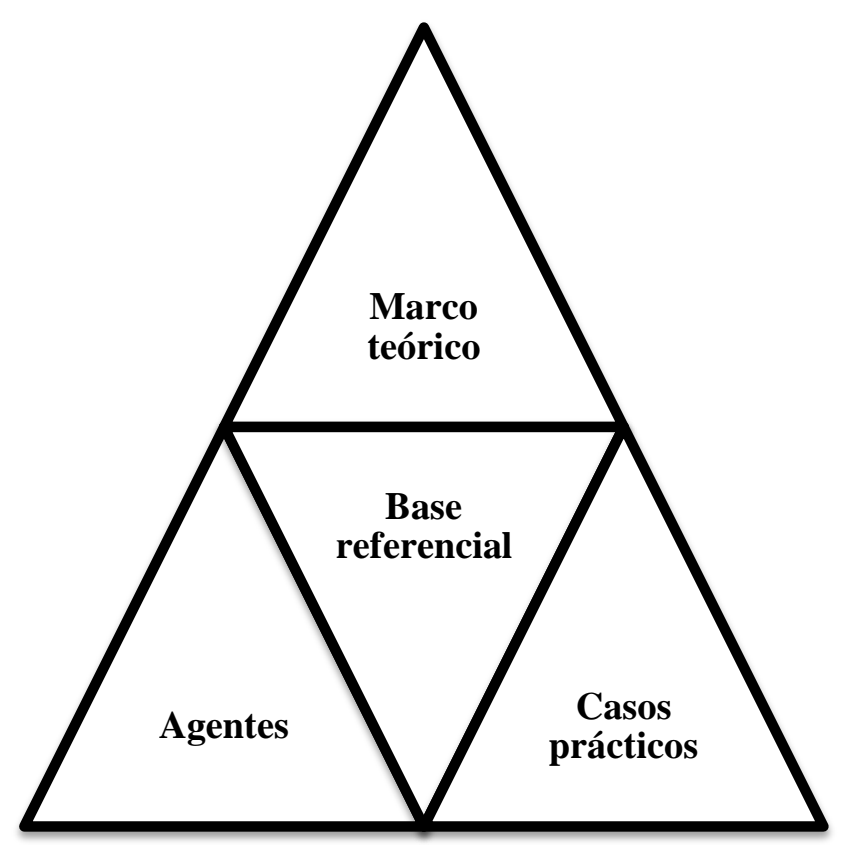

Figura 1. Representación conceptual de la propuesta docente

La propuesta docente aquí recogida evidencia, por una parte la escasez de desarrollo teórico-práctico que tanto el profesorado como el alumnado que gestionan documentos multilingües del ámbito ECOFIN europeo echan en falta y, por otra, la necesidad de una mayor armonización terminológica y conceptual en el ámbito europeo, sobre todo, tras el advenimiento de nuevas realidades ECOFIN en la legislación y entorno mediático de la Unión Europea.

Asimismo, lo anterior evidencia la existencia de una realidad conceptual propia e inherente a la Unión Europea desde el momento de su concepción como comunidad política, jurídica e institucional. El espacio compartido por los 28 Estados miembros actuales en donde afloran políticas comunes e instrumentos y procedimientos, que afectan en su mayoría a las 
legislaciones nacionales, genera conceptos propios en las distintas lenguas oficiales. Esta realidad, a la que denomino "eurolecto", precisa de un mayor reconocimiento así como armonización terminológica. En este sentido, son los propios profesionales que trabajan en los servicios lingüísticos de las instituciones europeas los que llevan a cabo la gesta armonizadora, dentro de sus posibilidades y reclamando mayor respuesta institucional.

En definitiva, este artículo pretende evidenciar la especificidad de los documentos de la Unión Europea (multilingües, oficiales y "eurolectales") puesta en valor a través de la docencia y formación al alumnado de traducción, interpretación y terminología, ofreciéndoles una base traductológica que sirva de referencia desde diferentes perspectivas para que los alumnos y las alumnas entren en contacto con la materia, comprendan hasta cierto grado las dificultades o complejidades a las que pueden enfrentarse y sean conscientes del protagonismo que asumen al gestionar o traducir textos de esta tipología en la que existen ciertas carencias.

REFERENCIAS BIBLIOGRÁFICAS

BRACKENIERS, Eduard, "Europe without frontiers and the language challenge". En: Terminologie \& Traduction, 2, 1995, pp. 13-17.

CAStellano MaRTínez, José María, "Esbozo histórico y evolución de las principales herramientas terminológicas de la Unión Europea: de la fragmentación a la convergencia instrumental". En: Revista Linguae, 1, 2014, pp. 39-73.

GAMBIER, Yves, "Mouvances eurolinguistiques. Cas de la Finlande". En: Europe et traduction, 1998, pp. 295-304.

GofFIN, Roger, "L'eurolecte: oui, jargon communautaire: non". En: Meta, 39, 1994, pp. 636-642.

GONZÁLEZ, Luis, "Armonización de términos financieros: CDO, ABS, CDS, etc.". En: Punto y coma, Boletín de los traductores españoles de las instituciones de la Unión Europea, 130, 2012, pp. 2-4.

HEYNOLD, Christian, "L'Union européenne: Jardin d'Éden ou Tour de Babel?". En: Terminologie \& Traduction, 3, 1999, pp. 5-14.

JAKOBSON, Robert, "On linguistic Aspects of Translation". En: The Translation Studies Reader, 2000, pp. 138-143.

JANSEEN, Maarten; VAN CAMPENHOUDT, Marc, "Terminologie traductive et représentation des connaissances: l'usage des relations hyponymiques". En: Langages, 157, 2005, pp. 63-79.

KJ/ER, Anne Lise, "Legal Translation in the European Union. A Research Field in Need of a New Approach". En: Language and the Law: International Outlooks, 2007, pp. 69-95. 
KOSKINEN, Kaisa, "Institutional Illusions. Translating in the EU Commision". En: The Translator, 6-1, 2000, pp. 49-65.

MINGASSON, Jean-Paul, "The written form of ECU". En: Terminologie \& Traduction, 1, 1989, pp. 65-66.

NeWmark, Peter, A Textbook of Translation. Nueva York: Prentice-Hall International, 1988.

NIDA, Eugene Albert, Toward a Science of Translating with Special Reference to Principles and Procedures involved in Bible Translation. Leiden: Brill, 1964.

Reglamento Delegado (UE) n.․46/2012 de la Comisión, de 21 de marzo de 2012, por el que se completa el Reglamento (CE) n. 0 1060/2009 del Parlamento Europeo y del Consejo en lo que respecta a las normas técnicas de regulación sobre el contenido y el formato de los informes periódicos sobre los datos relativos a las calificaciones que deberán presentar las agencias de calificación crediticia a la Autoridad Europea de Valores y Mercados, DO L 140 de 30.5.2012, p. 2.

ROGER, Goffin, "L'eurolecte: oui, jargon communautaire: non”. En: Meta, 39, 1994, pp. 636-642.

ŠARCEVIC, Susan, "Preserving Multilingualism in an Enlarged European Union". En: Terminologie et Traduction, 2, 2011, pp. 37-38.

VAN ELS, Theodorus Johannes Maria, "The European Union, its Institutions and its Languages: Some Language Political Observations". En: Current Issues in Language Planning, 4-2, 2001, pp. 311-360.

VV.AA. (Consejo de Asuntos Económicos y Financieros (Ecofín)). Disponible en línea en: http://www.consilium.europa.eu/es/councileu/configurations/ecofin/ [Fecha de consulta: 3 de junio de 2015].

VV.AA. (El euro). Disponible en línea en: http://europa.eu/about-eu/basic-information/money/euro/index es.htm [Fecha de consulta: 3 de junio de 2015].

VV.AA. (Eurogrupo). Disponible en línea en: http://www.consilium.europa.eu/es/council-eu/eurogroup/. [Fecha de consulta: 3 de junio de 2015].

VV.AA. (Eurosistema). Disponible en línea en: http://www.bde.es/bde/es/secciones/eurosistema/ [Fecha de consulta: 3 de junio de 2015].

VV.AA. (Fondo Europeo de Estabilidad Financiera (FEEF). Disponible en línea en: http://www.efsf.europa.eu/about/index.htm [Fecha de consulta: 3 de junio de 2015].

VV.AA. (Fundéu). "Zona del euro, zona euro y eurozona con inicial minúscula", 2009. Disponible en línea en: 
http://www.fundeu.es/recomendacion/zona-del-euro-y-euribor-formascorrectas-481/

[Fecha de consulta: 3 de junio de 2015].

VV.AA. (Grupo de Coordinación, Calidad y Terminología). "Glosario de términos y expresiones sobre la crisis económica Primera entrega". En: Punto y coma, Boletín de los traductores españoles de las instituciones de la Unión Europea, 130, 2012, pp. 2-4.

VV.AA. (Instrumentos financieros de la Unión Europea). Disponible en línea en:http://www.mineco.gob.es/portal/site/mineco/menuitem.b6c80362d 9873d0a91b0240e026041a0/?vgnextoid=e32f7cb59784c310VgnVCM 1000001d04140aRCRD

[Fecha de consulta: 3 de junio de 2015].

VV.AA. (Mecanismo Europeo de Estabilidad (MEDE)). Disponible en línea en: http://www.esm.europa.eu/index.htm

[Fecha de consulta: 3 de junio de 2015].

VV.AA. (Mecanismo Europeo de Estabilización Financiera (MEEF)). Disponible en línea en:

http://ec.europa.eu/economy finance/eu borrower/efsm/index en.htm Fecha de consulta: 3 de junio de 2015]. 